\title{
Global existence and asymptotic behavior of smooth solutions for a bipolar Euler-Poisson system in the quarter plane
}

Yeping Li

Correspondence: ypleemei@yahoo. com.cn

Department of Mathematics, Shanghai Normal University Shanghai 200234, P. R. China

\begin{abstract}
In the article, a one-dimensional bipolar hydrodynamic model (Euler-Poisson system) in the quarter plane is considered. This system takes the form of Euler-Poisson with electric field and frictional damping added to the momentum equations. The global existence of smooth small solutions for the corresponding initial-boundary value problem is firstly shown. Next, the asymptotic behavior of the solutions towards the nonlinear diffusion waves, which are solutions of the corresponding nonlinear parabolic equation given by the related Darcy's law, is proven. Finally, the optimal convergence rates of the solutions towards the nonlinear diffusion waves are established. The proofs are completed from the energy methods and Fourier analysis. As far as we know, this is the first result about the optimal convergence rates of the solutions of the bipolar Euler-Poisson system with boundary effects towards the nonlinear diffusion waves.

Mathematics Subject Classification: 35M20; 35Q35; 76W05.
\end{abstract}

Keywords: bipolar hydrodynamic model, nonlinear diffusion waves, smooth solutions, energy estimates

\section{Introduction}

In this note, we consider a bipolar hydrodynamic model (Euler-Poisson system) in one space dimension. Denoting by $n_{i}, j_{i}, P_{i}\left(n_{i}\right), i=1,2$, and $E$ the charge densities, current densities, pressures and electric field, the scaled equations of the hydrodynamic model are given by

$$
\left\{\begin{array}{l}
n_{1 t}+j_{1 x}=0 \\
j_{1 t}+\left(\frac{j_{1}^{2}}{n_{1}}+P_{1}\left(n_{1}\right)\right)_{x}=n_{1} E-\frac{j_{1}}{\tau_{1}} \\
n_{2 t}+j_{2 x}=0 \\
j_{2 t}+\left(\frac{j_{2}^{2}}{n_{2}}+P_{2}\left(n_{2}\right)\right)_{x}=-n_{2} E-\frac{j_{2}}{\tau_{2}} \\
\lambda^{2} E_{x}=n_{1}-n_{2} .
\end{array}\right.
$$

The positive constants $\tau_{i}(i=1,2)$ and $\lambda$ denote the relaxation time and the Debye length, respectively. The relaxation terms describe in a very rough manner the damping effect of a possible neutral background charge. The Debye length is related to the Coulomb screening of the charged particles. The hydrodynamic models are generally 
used in the description of charged particle fluids. These models take an important place in the fields of applied and computational mathematics. They can be derived from kinetic models by the moment method. For more details on the semiconductor applications, see $[1,2]$ and on the applications in plasma physics, see $[1,3]$. To begin with, we assume in the present article that the pressure-density functions satisfy

$$
P_{1}(n)=P_{2}(n)=n^{\gamma}, \quad \gamma \geq 1,
$$

and set $\tau_{1}, \tau_{2}$ and $\lambda$ to be one for simplicity In particular, we note that $\gamma=1$ is an important case in the applications of engineer. Hence, (1.1) can be simplifies as

$$
\left\{\begin{array}{l}
n_{1 t}+j_{1 x}=0 \\
j_{1 t}+\left(\frac{j_{1}^{2}}{n_{1}}+P\left(n_{1}\right)\right)_{x}=n_{1} E-j_{1}, \\
n_{2 t}+j_{2 x}=0 \\
j_{2 t}+\left(\frac{j_{2}^{2}}{n_{2}}+P\left(n_{2}\right)\right)_{x}=-n_{2} E-j_{2}, \\
E_{x}=n_{1}-n_{2} .
\end{array}\right.
$$

Recently, many efforts were made for the bipolar isentropic hydrodynamic equations of semiconductors. More precisely, Zhou and Li [4] and Tsuge [5] discussed the unique existence of the stationary solutions for the one-dimensional bipolar hydrodynamic model with proper boundary conditions. Natalini [6], and Hsiao and Zhang $[7,8]$ established the global entropic weak solutions in the framework of compensated compactness on the whole real line and spatial bounded domain respectively. Zhu and Hattori [9] proved the stability of steady-state solutions for a recombined bipolar hydrodynamical model. Ali and Jüngel [10] studied the global smooth solutions of Cauchy problem for multidimensional hydrodynamic models for two-carrier plasma. Lattanzio [11] and Li [12] studied the relaxation time limit of the weak solutions and local smooth solutions for Cauchy problems to the bipolar isentropic hydrodynamic models, respectively. Gasser and Marcati [13] discussed the relaxation limit, quasineutral limit and the combined limit of weak solutions for the bipolar Euler-Poisson system. Gasser et al. [14] investigated the large time behavior of solutions of Cauchy problem to the bipolar model basing on the fact that the frictional damping will cause the nonlinear diffusive phenomena of hyperbolic waves, while Huang and Li recently studied large-time behavior and quasineutral limit of $L^{\infty}$ solution of the Cauchy problem in [15]. As far as we know, no results about the global existence and large time behavior to (1.2) with boundary effect can be found. In this article we will consider global existence and asymptotic behavior of smooth solutions to the initial boundary value problems for the bipolar Euler-Poisson system on the quarter plane $\mathbb{R}_{+} \times \mathbb{R}_{+}$. Then, we now prescribe the initial-boundary value conditions:

$$
\left(n_{1}, j_{1}, n_{2}, j_{2}\right)(x, 0)=\left(n_{10}, j_{10}, n_{20}, j_{20}\right)(x) \rightarrow\left(n_{+}, j_{+}, n_{+}, j_{+}\right) \quad \text { as } \quad x \rightarrow+\infty,
$$

and

$$
j_{1}(0, t)=j_{2}(0, t)=0=E(0, t) .
$$

Moreover, we also investigate the time-asymptotic behavior of the solutions to (1.2)(1.4). Our results discussed below show that even for the case with boundary condition, the solutions of (1.2)-(1.4) can be captured by the corresponding porous equation 
as in initial data case. For the sake of simplicity, we can assume $j_{+}=0$. This assumption can be removed because of the exponential decay of the momentum at $x= \pm \infty$ induced by the linear frictional damping.

Finally, set

$$
\left(\varphi_{i 0}, z_{i 0}\right):=\left(-\int_{x}^{\infty}\left(n_{i 0}(\gamma)-\bar{n}_{i}\left(y+x_{i 0}, 0\right)\right) d y, j_{i 0}(x)-\bar{j}_{i}(x, 0)\right),
$$

here the nonlinear diffusion waves $\left(\bar{n}_{1}, \bar{j}_{1}, \bar{n}_{2}, \bar{j}_{2}\right)$ will be defined in Section 2, and the shift $x_{i 0}$ satisfy

$$
\int_{0}^{\infty}\left(n_{i 0}(x)-\bar{n}_{i}\left(x+x_{i 0}, t=0\right)\right) d x=0,
$$

which can be computed from the standard arguments, see [16-19].

Throughout this article $C$ always denotes a harmless positive constant. $L^{p}\left(\mathbb{R}_{+}\right)$is the space of square integrable real valued function defined on $\mathbb{R}_{+}$with the norm $\|\cdot\|_{L^{p}\left(\mathbb{R}^{+}\right)}$ and $H^{k}\left(\mathbb{R}_{+}\right)$denotes the usual Sobolev space with the norm $\|\cdot\|_{k}$.

Now one of main results in this paper is stated as follows.

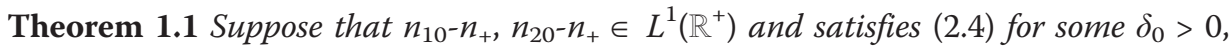
$\left(\phi_{10}, z_{10}, \phi_{20}, z_{20}\right) \in\left(H^{3}\left(\mathbb{R}^{+}\right) \cap L^{1}\left(\mathbb{R}_{+}\right)\right) \times\left(H^{2}\left(\mathbb{R}_{+}\right) \cap L^{1}\left(\mathbb{R}_{+}\right)\right) \times\left(H^{3}\left(\mathbb{R}^{+}\right) \cap L^{1}\left(\mathbb{R}_{+}\right)\right) \times\left(H^{2}\right.$ $\left.\left(\mathbb{R}_{+}\right) \cap L^{1}\left(\mathbb{R}_{+}\right)\right)$with $x_{10}=x_{20}$ and that

$$
\begin{aligned}
& \left\|n_{10}-n_{+}\right\|_{L^{1}\left(\mathbb{R}_{+}\right)}+\left\|n_{20}-n_{+}\right\|_{L^{1}\left(\mathbb{R}_{+}\right)}+\left\|\left(\varphi_{10}, \varphi_{20}\right)\right\|_{H^{3}\left(\mathbb{R}^{+}\right)}+\left\|\left(z_{10}, z_{20}\right)\right\|_{H^{2}\left(\mathbb{R}^{+}\right)} \\
+ & \left\|\varphi_{10}, \varphi_{20}\right\|_{L^{1}\left(\mathbb{R}_{+}\right)}+\left\|\left(z_{10}, z_{20}\right)\right\|_{L^{1}\left(\mathbb{R}_{+}\right)}+\delta_{0} \ll 1
\end{aligned}
$$

hold. Then there exists a unique time-global solution $\left(n_{1}, j_{1}, n_{2}, j_{2}\right)(x, t)$ of IBVP (1.2)(1.4), such that for $i=1,2$,

$$
\begin{aligned}
& n_{i}-\bar{n}_{i} \in C^{k}\left(0, \infty, H^{2-k}\left(\mathbb{R}_{+}\right)\right), \quad k=0,1,2, \\
& j_{i}=\bar{j}_{i} \in C^{k}\left(0, \infty, H^{1-k}\left(\mathbb{R}_{+}\right)\right), \quad k=0,1, \\
& E \in C^{k}\left(0, \infty, H^{3-k}\left(\mathbb{R}_{+}\right)\right), \quad k=0,1,2,3,
\end{aligned}
$$

and

$$
\begin{aligned}
& \left\|\partial_{x}^{k}\left(n_{1}-\bar{n}_{1}, n_{2}-\bar{n}_{2}\right)\right\|_{L^{2}\left(\mathbb{R}_{+}\right)} \leq C(1+t)^{-\frac{k}{2}, \quad k=0,1,2,} \\
& \left\|\partial_{x}^{k}\left(j_{1}-\bar{j}_{1}, j_{2}-\bar{j}_{2}\right)\right\|_{L^{2}\left(\mathbb{R}_{+}\right)} \leq C(1+t)^{-\frac{k+2}{2}}, \quad k=0,1,2, \\
& \left\|\partial_{x}^{k} E\right\|_{L^{2}\left(\mathbb{R}_{+}\right)} \leq C e^{-\alpha t}, \quad k=0,1,2
\end{aligned}
$$

where $\alpha>0$ and $C$ is positive constant.

Next, with the help of Fourier analysis, we can obtain the following optimal convergence rate. 
Theorem 1.2 Under the assumptions of Theorem 1.1, it holds that

$$
\begin{aligned}
& \left\|\partial_{x}^{k}\left(n_{1}-\bar{n}_{1}, n_{2}-\bar{n}_{2}\right)\right\|_{L^{2}\left(\mathbb{R}_{+}\right)} \leq C(1+t)^{-\frac{2 k+3}{4}}, \quad k=0,1, \\
& \left\|\left(n_{1}-\bar{n}_{1}, n_{2}-\bar{n}_{2}\right)\right\|_{L^{p}\left(\mathbb{R}_{+}\right)} \leq C(1+t)^{-\frac{2 p-1}{2 p}}, \quad 2 \leq p \leq+\infty, \\
& \left\|\left(j_{1}-\bar{j}_{1}, j_{2}-\bar{j}_{2}\right)\right\|_{L^{2}\left(\mathbb{R}_{+}\right)} \leq C(1+t)^{-\frac{5}{4}} .
\end{aligned}
$$

Remark 1.3 The condition (2.4) implies

$$
\int_{0}^{\infty}\left(n_{10}(x)-n_{20}(x)\right) d x=0
$$

and it is a technique one. As to more general case, we will discuss it in the forthcoming future. Theorems 1.1 and 1.2 show that the nonlinear diffusive phenomena is maintained in the bipolar Euler-Poisson system with the interaction of two particles and the additional electric field, which indeed implies that this diffusion effect is essentially due to the friction of momentum relaxation.

Using the energy estimates, we can establish a priori estimate, which together with local existence, leads to global existence of the smooth solutions for IBVP (1.2)-(1.4) by standard continuity arguments. In order to obtain the asymptotic behavior and optimal decay rate, noting that $E=\phi_{1}-\phi_{2}$ satisfies the damping "Klein-Gordon" equation (see $[14,15]$ ), we first obtain the exponential decay rate of the electric field $E$ by energy methods. Then, we can establish the algebraical decay rate of the perturbed densities $\phi_{1}$ and $\phi_{2}$. Finally, from the estimates of the wave equation with damping in [20] and using the idea of [16], we show the optimal algebraical decay rates of the total perturbed density $\phi_{1}+\phi_{2}$, which together with the exponential decay rate of the difference of two perturbed densities, yields the optimal decay rate. In these procedure, we have overcome the difficulty from the coupling and cancelation interaction between $n_{1}$ and $n_{2}$. Finally, it is worth mentioning that similar results about the Euler equations with damping have been extensively studied by many authors, i.e., the authors of [16-19,21,22], etc.

The rest of this article is arranged as follows. We first construct the optimal nonlinear diffusion waves and recall some inequalities in Section 2. In Section 3, we reformulate the original problem, and show the main Theorem. Section 4 is to prove an important decay estimate, which has been used to show the main theorem in Section 3.

\section{The nonlinear diffusion waves}

In this section, we first construct the optimal nonlinear diffusion waves of (1.2) in the quarter plane. To begin with, we define our diffusion waves as

$$
\bar{n}_{i}=n_{+}+\delta_{0} \phi(x, t+1), \quad \bar{j}_{1}=-P\left(\bar{n}_{i}\right)_{x}, \quad i=1,2 .
$$

Here the function $\varphi(x, t+1)$ (here using $t+1$ instead of $t$ is to avoid the singularity of solution decay at the point $t=0$ ) solves

$$
\delta_{0} \phi_{t}-P\left(n_{+}+\delta_{0} \phi\right)_{x x}=0, \quad(x, t) \in \mathbb{R}_{+} \times \mathbb{R}_{+}
$$


namely,

$$
\phi_{t}-P^{\prime}\left(n_{+}\right) \phi_{x x}=\frac{1}{\delta_{0}}\left(P\left(n_{+}+\delta_{0} \phi-P\left(n_{+}\right)-P^{\prime}\left(n_{+}\right) \phi\right)_{x x^{\prime}} \quad(x, t) \in \mathbb{R}_{+} \times \mathbb{R}_{+},\right.
$$

with the initial boundary values

$$
\left.\phi_{x}\right|_{x=0}=0,\left.\quad \phi\right|_{x=+\infty}=0,\left.\quad \phi\right|_{t=0}=\phi(x, 1)=: \phi_{0}(x) .
$$

Where $\varphi_{0}(x)$ is a given smooth function such that

$$
\phi_{0}(x) \in L^{1}\left(\mathbb{R}_{+}\right) \text {and } \int_{0}^{\infty} \phi_{0}(x) d x \neq 0,
$$

and $\delta_{0}$ is a constant satisfying

$$
\int_{0}^{\infty}\left(n_{i 0}(x)-n_{+}\right) d x-\delta_{0} \int_{0}^{\infty} \phi_{0}(x) d x=0 .
$$

Note that from the assumptions in Theorem 1.1 and (2.3), there exists $\delta_{0}$ satisfies (2.4).

The existence of $\varphi(x, t)$ has been shown in [16], and the following estimates of $\varphi(x, t)$ hold:

$$
\begin{aligned}
& \left\|\partial_{t}^{j} \partial_{x}^{k} \phi\right\|_{L^{2}\left(\mathbb{R}_{+}\right)} \leq C \delta_{0}(1+t)^{-(4 j+2 k+1) / 4}, \\
& \left\|\phi_{x t}\right\|_{L^{1}\left(\mathbb{R}_{+}\right)} \leq C \delta_{0}(1+t)^{-3 / 2}
\end{aligned}
$$

with the help of the Green function method and energy estimates.

Then $\left(n_{1}, j_{1}, n_{2}, j_{2}\right)(x, t)$ is the required nonlinear diffusion wave, and satisfies

$$
\bar{n}_{i t}+\bar{j}_{i x}=0, \quad \bar{j}_{i}=-P\left(\bar{n}_{i}\right)_{x \prime}
$$

with the boundary restrictions

$$
\left.\bar{n}_{i x}\right|_{x=0}=0,\left.\quad n_{i}\right|_{x=+\infty}=n_{+} .
$$

From (2.5) and (2.6), we have

Lemma 2.1 If $\left(\bar{n}_{i}, \bar{j}_{i}\right)(x, t)$ is defined as above, then

$$
\begin{aligned}
& \left\|\partial_{t}^{l} \partial_{x}^{k}\left(\bar{n}_{i}-n_{+}\right)\right\|_{L^{2}\left(\mathbb{R}_{+}\right)} \leq C \delta_{0}(1+t)^{-(4 l+2 k+1) / 4}, \\
& \left\|\bar{n}_{i x t}\right\|_{L^{1}\left(\mathbb{R}_{+}\right)} \leq C \delta_{0}(1+t)^{-3 / 2} .
\end{aligned}
$$

Next, we introduce some inequalities of Sobolev type.

Lemma 2.2 The following inequalities hold

$$
\|f\|_{L^{p}\left(\mathbb{R}_{+}\right)} \leq C\|f\|_{1^{\prime}} \quad p \in[2, \infty]
$$

for some constant $C>0$.

Finally, for later use, we also need 
Lemma 2.3 [20]Assume that $K_{i}(x, t)(i=0,1)$ are the fundamental solutions of

$$
K_{i t t}+K_{i t}-K_{i x x}=0, \quad i=0,1
$$

with

$$
K_{0}(x, 0)=\delta(x), \quad K_{1}(x, 0)=0, \quad \frac{d}{d t} K_{0}(x, 0)=0, \quad \frac{d}{d t} K_{1}(x, 0)=\delta(x),
$$

where $\delta(x)$ is the Delta function.

If $f \in L^{1}\left(\mathbb{R}_{+}\right) \cap H^{j+k-1}\left(\mathbb{R}_{+}\right)$, then

$$
\begin{aligned}
& \left\|\partial_{t}^{j} \partial_{x}^{k} \int_{0}^{\infty}\left(K_{1}(x-y, t)-K_{1}(x+y, t)\right) f(y) d y\right\|_{L^{2}\left(\mathbb{R}_{+}\right)} \\
& \leq C(1+t)^{-j-\frac{2 k+1}{4}}\left(\|f\|_{L^{1}\left(\mathbb{R}_{+}\right)}+\|f\|_{j+k-1}\right) .
\end{aligned}
$$

If $f \in L^{1}\left(\mathbb{R}_{+}\right) \cap H^{j+k}\left(\mathbb{R}_{+}\right)$, then

$$
\begin{aligned}
& \left\|\partial_{t}^{j} \partial_{x}^{k} \int_{0}^{\infty}\left(K_{0}(x-y, t)-K_{0}(x+y, t)\right) f(y) d y\right\|_{L^{2}\left(\mathbb{R}_{+}\right)} \\
& \leq C(1+t)^{-j-\frac{2 k+1}{4}}\left(\|f\|_{L^{1}\left(\mathbb{R}_{+}\right)}+\|f\|_{j+k}\right) .
\end{aligned}
$$

\section{Global existence and algebraical decay rate}

In this section we are going to reformulate the original problem and establish the global existence and algebraical decay rate. To begin with, from (1.2) and (2.7), we notice that

$$
\int_{0}^{\infty}\left(n_{i}-\bar{n}_{i}\right)(x, t)=\int_{0}^{\infty}\left(n_{i 0}-n_{+}\right) d x-\delta_{0} \int_{0}^{\infty} \phi_{0}(x) d x=0, \quad i=1,2 .
$$

Thus, it is reasonable to introduce the following perturbations as our new variables

$$
\varphi_{i}=-\int_{x}^{\infty}\left(n_{i}-\bar{n}_{i}\right)(y, t) d y, z_{i}=j_{i}-\bar{j}_{i}
$$

which yields

$$
\left\{\begin{array}{l}
\varphi_{1 t}+z_{1}=0, \\
z_{1 t}+\left(\frac{\left(z_{1}+\bar{j}_{1}\right)^{2}}{\bar{n}_{1}+\varphi_{1 x}}+P\left(\bar{n}_{1}+\varphi_{1 x}\right)-P\left(\bar{n}_{1}\right)\right)_{x}+z_{1}=\left(\bar{n}_{1}+\varphi_{1 x}\right) E+P\left(\bar{n}_{1}\right)_{x t^{\prime}} \\
\varphi_{2 t}+z_{2}=0 \\
z_{2 t}+\left(\frac{\left(z_{2}+\bar{j}_{2}\right)^{2}}{\bar{n}_{2}+\varphi_{2 x}}+P\left(\bar{n}_{2}+\varphi_{2 x}\right)-P\left(\bar{n}_{2}\right)\right)_{x}+z_{2}=-\left(\bar{n}_{2}+\varphi_{2 x}\right) E+P\left(\bar{n}_{2}\right) x_{t} \\
\varphi_{2 t}+z_{2}=0, \\
E=\varphi_{1}-\varphi_{2} \\
\left(\varphi_{1}, z_{1}, \varphi_{2}, z_{2}\right)_{t=0}=\left(\varphi_{10}, z_{10}, \varphi_{10}, z_{10}\right)(x) \\
\left.\left(\varphi_{1}, \varphi_{2}\right)\right|_{x=0}=0 .
\end{array}\right.
$$


Further, we have

$$
\begin{aligned}
& \varphi_{1 t t}-\left(P\left(\bar{n}_{1}+\varphi_{1 x}\right)-P\left(\bar{n}_{1}\right)\right)_{x}+\varphi_{1 t}+\left(\bar{n}_{1}+\varphi_{1 x}\right) E=P\left(\bar{n}_{1}\right)_{x t}+f_{1 x} \\
& \varphi_{2 t t}-\left(P\left(\bar{n}_{2}+\varphi_{2 x}\right)-P\left(\bar{n}_{2}\right)\right)_{x}+\varphi_{2 t}-\left(\bar{n}_{2}+\varphi_{2 x}\right) E=P\left(\bar{n}_{2}\right)_{x t}+f_{1 x}
\end{aligned}
$$

and

$$
E_{t t}+E_{t}+\left(\bar{n}_{1}+\bar{n}_{2}\right) E=f_{3},
$$

here

$$
\begin{aligned}
f_{i}= & \frac{\left(\varphi_{i t}+P\left(\bar{n}_{1}\right) x\right)^{2}}{\bar{n}_{i}+\varphi_{i x}}, \quad i=1,2 \\
f_{3}= & -P\left(\bar{n}_{1}\right)_{x t}+P\left(\bar{n}_{2}\right)_{x t}+\left(P\left(\bar{n}_{1}+\varphi_{1 x}\right)-P\left(\bar{n}_{1}\right)\right)_{x} \\
& -\left(P\left(\bar{n}_{2}+\varphi_{2 x}\right)-P\left(\bar{n}_{2}\right)\right)_{x}-\left(\varphi_{1 x}+\varphi_{2 x}\right) E+\left(f_{1}-f_{2}\right)_{x} .
\end{aligned}
$$

Next, by the standard continuous arguments, we can obtain the global existence of smooth solutions. That is, we combine the local existence and a priori estimate. For the local existence of the solution to (3.2)-(3.3), we see, e.g., [20] and references therein. In the following we devote ourselves to the a priori estimates of the solution $\left(\phi_{1}, \phi_{2}, E\right)(0<t<T)$ to $(3.2)-(3.3)$ under the a priori assumption

$$
N(T):=\sup _{0<t<T}\left((1+t)^{k}\left\|\partial_{x}^{k}\left(\varphi_{1}, \varphi_{2}\right)\right\|_{L^{2}\left(\mathbb{R}_{+}\right)}^{2}+(1+t)^{k+2}\left\|\partial_{x}^{k}\left(\varphi_{1 t}, \varphi_{2 t}\right)\right\|_{L^{2}\left(\mathbb{R}_{+}\right)}^{2}\right) \ll 1 .
$$

Noting

$$
\varphi_{i}(0, t)=\varphi_{i t}(0, t)=\varphi_{i x x}(0, t)=\varphi_{i t x x}(0, t)=0, \quad i=1,2,
$$

we can obtain the following estimates by using a similar argument of [14]. Since the proof is tedious but similar as in the previous works, we only list the results and omit its details.

Lemma 3.1 For $T>0$, let $\left(\phi_{1}, \phi_{2}, E\right)(x, t)$ be the solution to (3.2)-(3.3). Then, it holds for $N(T)+\delta_{0}$ that

$$
\begin{aligned}
& \left\|\left(\varphi_{1}, \varphi_{2}\right)\right\|_{3}^{2}+\left\|\left(\varphi_{1 t}, \varphi_{2 t}, E\right)\right\|_{3}^{2}+\int_{0}^{T}\left\|\left(\varphi_{1 x}, \varphi_{2 x}, \varphi_{1 t}, \varphi_{2 t}, E\right)\right\|_{2}^{2} d t \\
\leq & C\left(\left\|\varphi_{10}, \varphi_{20}\right\|_{3}^{2}+\left\|\left(z_{10}, z_{20}\right)\right\|_{2}^{2}+\delta_{0}\right) .
\end{aligned}
$$

Lemma 3.2 For $T>0$, let $\left(\phi_{1}, \phi_{2}, E\right)(x, t)$ be the solution to (3.2)-(3.3). Then, it holds for $N(T)+\delta_{0}$ that

$$
\left\|\left(E, E_{x}, E_{t}, E_{x x}, E_{x t}, E_{t t}\right)\right\|^{2} \leq C\left(\left\|\varphi_{10}, \varphi_{20}\right\|_{3}^{2}+\left\|\left(z_{10}, z_{20}\right)\right\|_{2}^{2}+\delta_{0}\right) e^{-\beta t},
$$

for some positive constant $\beta$.

Lemma 3.3 For $T>0$, let $\left(\phi_{1}, \phi_{2}, E\right)(x, t)$ be the solution to (3.2)-(3.3). Then there exist positive constants $C$ such that

$$
\left\|\partial_{x}^{k}\left(\varphi_{1}, \varphi_{2}\right)\right\|_{L^{2}\left(\mathbb{R}_{+}\right)} \leq C(1+t)^{-\frac{k}{2}}, \quad k=0,1,2,3,
$$




$$
\left\|\partial_{x}^{k}\left(z_{1}, z_{2}\right)\right\|_{L^{2}\left(\mathbb{R}_{+}\right)} \leq C(1+t)^{-\frac{k+2}{2}}, \quad k=0,1,2,
$$

and

$$
\begin{aligned}
& (1+t)^{2}\left\|z_{1 t}, z_{2 t}\right\|_{L^{2}\left(\mathbb{R}_{+}\right)} \\
+ & (1+t)^{-\frac{5}{2}}\left(\left\|\left(z_{1 x t}, z_{2 x t}\right)\right\|_{L^{2}\left(\mathbb{R}_{+}\right)}+\left\|\left(z_{1 t t}, z_{2 t t}\right)\right\|_{L^{2}\left(\mathbb{R}_{+}\right)}\right) \leq C .
\end{aligned}
$$

In conclusion, we have

Theorem 3.4 Under the assumptions in Theorem 1.1, there exists a unique time global solution $\left(\phi_{1}, z_{1}, \phi_{2}, z_{2}\right)$ of the IBVP (3.1) such that

$$
\begin{aligned}
& \varphi_{1}, \varphi_{2}, E \in C^{k}\left(0, \infty, H^{3-k}\left(\mathbb{R}_{+}\right)\right), \quad k=0,1,2,3, \\
& z_{1}, z_{2} \in C^{k}\left(0, \infty, H^{2-k}\left(\mathbb{R}_{+}\right)\right), \quad k=0,1,2,
\end{aligned}
$$

and there exist positive constants $C, \alpha$ such that

$$
\begin{aligned}
& \left\|\partial_{x}^{k}\left(\varphi_{1}, \varphi_{2}\right)\right\|_{L^{2}\left(\mathbb{R}_{+}\right)} \leq C(1+t)^{-\frac{k}{2}, \quad k=0,1,2,3,} \\
& \left\|\partial_{x}^{k}\left(z_{1}, z_{2}\right)\right\|_{L^{2}\left(\mathbb{R}_{+}\right)} \leq C(1+t)^{-\frac{k+2}{2}}, \quad k=0,1,2, \\
& \left\|\left(E, E_{x}, E_{t}, E_{x t}, E_{x x}\right)\right\|_{L^{2}\left(\mathbb{R}_{+}\right)} \leq C e^{-\alpha t},
\end{aligned}
$$

and

$$
\begin{aligned}
& (1+t)^{2}\left\|z_{1 t}, z_{2 t}\right\|_{L^{2}\left(\mathbb{R}_{+}\right)} \\
+ & (1+t)^{-\frac{5}{2}}\left(\left\|\left(z_{1 x t}, z_{2 x t}\right)\right\|_{L^{2}\left(\mathbb{R}_{+}\right)}+\left\|\left(z_{1 t t}, z_{2 t t}\right)\right\|_{L^{2}\left(\mathbb{R}_{+}\right)}\right) \leq C .
\end{aligned}
$$

\section{The optimal convergence rate}

In this section we are going to show the optimal decay rate. First of all, we improve the decay rates in Theorem 3.4 to be optimal as follows.

Proposition 4.1 Under the assumptions in Theorem 1.1, the solution $\left(\phi_{1}, z_{1}, \phi_{2}, z_{2}\right)$ decay time asymptotically as

$$
\begin{aligned}
& \left\|\partial_{x}^{k}\left(\varphi_{1}, \varphi_{2}\right)\right\|_{L^{2}\left(\mathbb{R}_{+}\right)} \leq C(1+t)^{-\frac{2 k+1}{4}}, \quad k=0,1,2, \\
& \left\|\left(z_{1}, z_{2}\right)\right\|_{L^{2}\left(\mathbb{R}_{+}\right)} \leq C(1+t)^{-\frac{5}{4}} .
\end{aligned}
$$

Based on the above Proposition, we can immediately prove Theorem 1.2.

Proof of Theorem 1.2 Thanks to Proposition 4.1, and by noting that $\varphi_{i x}=n_{i}-\bar{n}_{i}, z_{i}=j_{i}-\bar{j}_{i}$, we have 


$$
\begin{aligned}
\left\|\partial_{x}^{k}\left(n_{1}-\bar{n}_{1}, n_{2}-\bar{n}_{2}\right)(t)\right\|_{L^{2}\left(\mathbb{R}_{+}\right)} & =\left\|\partial_{x}^{k+1}\left(\varphi_{1}, \varphi_{2}\right)(t)\right\|_{L^{2}\left(\mathbb{R}_{+}\right)} \\
& \leq\left\|\partial_{x}^{k+1}\left(\varphi_{1}, \varphi_{2}\right)(t)\right\|_{L^{2}\left(\mathbb{R}_{+}\right)} \\
& \leq C(1+t)^{-\frac{2(k+1)+1}{4}}, \quad k=0,1,
\end{aligned}
$$

and

$$
\begin{aligned}
\left\|\left(j_{1}-\bar{j}_{1}, j_{2}-\bar{j}_{2}\right)(t)\right\|_{L^{2}\left(\mathbb{R}_{+}\right)} & =\left\|\left(z_{1}, z_{2}\right)(t)\right\|_{L^{2}\left(\mathbb{R}_{+}\right)} \\
& \leq C(1+t)^{-\frac{5}{4}} .
\end{aligned}
$$

This proved (1.8) and (1.10). Next, using Lemma 2.2, we get

$$
\begin{aligned}
\left\|n_{1}-\bar{n}_{1}\right\|_{L^{p}\left(\mathbb{R}_{+}\right)} & \leq\left\|n_{1}-\bar{n}_{1}\right\|_{L^{\infty}\left(\mathbb{R}_{+}\right)}^{\frac{p-2}{p}}\left\|n_{1}-\bar{n}_{1}\right\|_{L^{2}\left(\mathbb{R}_{+}\right)}^{\frac{2}{p}} \\
& \leq\left(\sqrt{2}\left\|n_{1}-\bar{n}_{1}\right\|_{L^{2}\left(\mathbb{R}_{+}\right)}^{\frac{1}{2}}\left\|\partial_{x}\left(n_{1}-\bar{n}_{1}\right)\right\|_{L^{2}\left(\mathbb{R}_{+}\right)}^{\frac{1}{2}}\right)^{\frac{p-2}{p}}\left\|n_{1}-\bar{n}_{1}\right\|_{L^{2}\left(\mathbb{R}_{+}\right)}^{\frac{2}{p}} \\
& =2^{\frac{p-2}{2 p}}\left\|n_{1}-\bar{n}_{1}\right\|_{L^{2}\left(\mathbb{R}_{+}\right)}^{\frac{p-2}{2 p}+\frac{2}{p}}\left\|\partial_{x}\left(n_{1}-\bar{n}_{1}\right)\right\|_{L^{2}\left(\mathbb{R}_{+}\right)}^{\frac{p-2}{2 p}} \\
& \leq C(1+t)^{-\frac{3}{4} \times\left(\frac{p-2}{2 p}+\frac{2}{p}\right)}(1+t)^{-\frac{5}{4} \times \frac{p-2}{2 p}} \\
& \leq C(1+t)^{-\left(1-\frac{1}{2 p}\right)},
\end{aligned}
$$

and

$$
\begin{aligned}
\left\|n_{2}-\bar{n}_{2}\right\|_{L^{p}\left(\mathbb{R}_{+}\right)} & \leq\left\|n_{2}-\bar{n}_{2}\right\|_{L^{\infty}\left(\mathbb{R}_{+}\right)}^{\frac{p-2}{p}}\left\|n_{2}-\bar{n}_{2}\right\|_{L^{2}\left(\mathbb{R}_{+}\right)}^{\frac{2}{p}} \\
& \leq\left(\sqrt{2}\left\|n_{2}-\bar{n}_{2}\right\|_{L^{2}\left(\mathbb{R}_{+}\right)}^{\frac{1}{2}}\left\|\partial_{x}\left(n_{2}-\bar{n}_{2}\right)\right\|_{L^{2}\left(\mathbb{R}_{+}\right)}^{\frac{1}{2}}\right)^{\frac{p-2}{p}}\left\|n_{2}-\bar{n}_{2}\right\|_{L^{2}\left(\mathbb{R}_{+}\right)}^{\frac{2}{p}} \\
& =2^{\frac{p-2}{2 p}}\left\|n_{2}-\bar{n}_{2}\right\|_{L^{2}\left(\mathbb{R}_{+}\right)}^{\frac{p-2}{p}+\frac{2}{p}}\left\|\partial_{x}\left(n_{2}-\bar{n}_{2}\right)\right\|_{L^{2}\left(\mathbb{R}_{+}\right)}^{\frac{p-2}{2 p}} \\
& \leq C(1+t)^{-\frac{3}{4} \times\left(\frac{p-2}{2 p}+\frac{2}{p}\right)}(1+t)^{-\frac{5}{4} \times \frac{p-2}{2 p}} \\
& \leq C(1+t)^{-\left(1-\frac{1}{2 p}\right)} .
\end{aligned}
$$

This prove (1.9).

In the following we focus on the proof of Proposition 4.1. To begin with, we notice that

$$
\left(\varphi_{1}+\varphi_{2}\right)_{t t}-P^{\prime}\left(n_{+}\right)\left(\varphi_{1}+\varphi_{2}\right)_{x x}+\left(\varphi_{1}+\varphi_{2}\right)_{t}=F,
$$

with

$$
\begin{aligned}
F= & \left(P\left(\bar{n}_{1}+\varphi_{1 x}\right)-P\left(\bar{n}_{1}\right)-P^{\prime}\left(n_{+}\right) \varphi_{1 x}\right)_{x}+\left(P\left(\bar{n}_{2}+\varphi_{2 x}\right)-P\left(\bar{n}_{2}\right)-P^{\prime}\left(n_{+}\right) \varphi_{2 x}\right)_{x} \\
& -P\left(\bar{n}_{1}\right)_{x t}-P\left(\bar{n}_{2}\right)_{x t}-\left(\varphi_{1 x}+\varphi_{2 x}\right) E+\left(f_{1}+f_{2}\right)_{x} .
\end{aligned}
$$

Proof of Proposition 4.1. Firstly, we prove the optimal decay rates for $\left\|\partial_{x}^{k}\left(\varphi_{1}, \varphi_{2}\right)\right\|_{L^{2}}(k=0,1,2)$, namely, (4.1). By (4.3) and Lemma 2.3, we obtain 


$$
\begin{aligned}
& \left(\varphi_{1}+\varphi_{2}\right)(x, t) \\
= & \int_{0}^{\infty}\left(K_{0}(x-y, t)-K_{0}(x+y, t)\right)\left(\varphi_{10}+\varphi_{20}\right)(y) d y \\
& +\int_{0}^{\infty}\left(K_{1}(x-y, t)-K_{1}(x+y, t)\right)\left(z_{10}+z_{20}\right)(y) d y \\
& +\int_{0}^{t} \int_{0}^{\infty}\left(K_{1}(x-y, t-\tau)-K_{1}(x+y, t-\tau)\right) F(y, \tau) d y d \tau .
\end{aligned}
$$

By differentiating (4.4) $k$-times $(k=0,1,2)$ with respect to $x$, and by taking its $L^{2}(\mathbb{R}$ +)-norm, we obtain

$$
\begin{aligned}
& \left\|\partial_{x}^{k}\left(\varphi_{1}+\varphi_{2}\right)(t)\right\|_{L^{2}\left(\mathbb{R}_{+}\right)} \\
= & \left\|\partial_{x}^{k} \int_{0}^{\infty}\left(K_{0}(x-y, t)-K_{0}(x+y, t)\right)\left(\varphi_{10}+\varphi_{20}\right)(y) d y\right\|_{L^{2}\left(\mathbb{R}_{+}\right)} \\
& +\left\|\int_{0}^{\infty}\left(K_{1}(x-y, t)-K_{1}(x+y, t)\right)\left(z_{10}+z_{20}\right)(y) d y\right\|_{L^{2}\left(\mathbb{R}_{+}\right)}\|\|_{0} d \tau . \\
& +\int_{0}^{t}\left\|\partial_{x}^{k} \int_{0}^{\infty}\left(K_{1}(x-y, t-\tau)-K_{1}(x+y, t-\tau)\right) F(y, \tau) d y\right\|_{L^{2}\left(\mathbb{R}_{+}\right)} d \tau
\end{aligned}
$$

Since $\phi_{10}+\phi_{20} \in L^{1}\left(\mathbb{R}_{+}\right) \cap H^{3}\left(\mathbb{R}_{+}\right)$and $z_{10}+z_{20} \in L^{1}\left(\mathbb{R}_{+}\right) \cap H^{2}\left(\mathbb{R}_{+}\right)$, we apply Lemma 2.3 then to have

$$
\begin{aligned}
& \left\|\partial_{x}^{k} \int_{0}^{\infty}\left(K_{0}(x-y, t)-K_{0}(x+y, t)\right)\left(\varphi_{10}+\varphi_{20}\right)(y) d y\right\|_{L^{2}\left(\mathbb{R}_{+}\right)} \\
& \leq C\left(\left\|\varphi_{10}, \varphi_{20}\right\|_{L^{2}\left(\mathbb{R}_{+}\right)}+\left\|\varphi_{10}, \varphi_{20}\right\|_{3}\right)(1+t)^{-(2 k+1) / 4},
\end{aligned}
$$

and

$$
\begin{aligned}
& \left\|\int_{0}^{\infty}\left(K_{1}(x-y, t)-K_{1}(x+y, t)\right)\left(z_{10}+z_{20}\right)(y) d y\right\|_{L^{2}\left(\mathbb{R}_{+}\right)} \\
& \leq C\left(\left\|z_{10}, z_{20}\right\|_{L^{1}\left(\mathbb{R}_{+}\right)}+\left\|z_{10}, z_{20}\right\|_{2}\right)(1+t)^{-(2 k+1) / 4},
\end{aligned}
$$

for $k=0,1,2$.

Now we are going to estimate the last term in (4.5). By Taylor's expansion, and by noticing the definition of $F$, we have

$$
\begin{aligned}
|F| \sim & O(1)\left(\left|\bar{n}_{1 x} \bar{n}_{1 t}\right|+\left|\bar{n}_{1 x t}\right|+\left|\left(\left(\bar{n}_{1}-n_{+}\right) \varphi_{1 x}\right)_{x}\right|+\left|\left(\left(\bar{n}_{1}-n_{+}\right) \varphi_{2 x}\right)_{x}\right|\right. \\
& +\left|\hat{n}_{1 x}\right|+\left|\left(\varphi_{1 x}^{2}\right)_{x}\right|+\left|\left(\varphi_{2 x}^{2}\right)_{x}\right|+\left|\varphi_{1 x t} \varphi_{1 x}\right|+\left|\varphi_{1 t}^{2} \bar{n}_{1 x}\right|+\left|\varphi_{2 x t} \varphi_{2 x}\right|+\left|\varphi_{2 t}^{2} \bar{n}_{1 x}\right| \\
& +\left|\varphi_{1 t}^{2} \varphi_{1 x x}\right|+\left|\varphi_{2 t}^{2} \varphi_{2 x x}\right|+\left|\left(\varphi_{1}+\varphi_{2}\right)_{x} E\right|,
\end{aligned}
$$


and

$$
\begin{aligned}
\left|\partial_{x}^{k} F\right| \sim & O(1)\left(\left|\partial_{x}^{k} \bar{n}_{1 x} \bar{n}_{1 t}\right|+\left|\partial_{x}^{k} \bar{n}_{1 x t}\right|+\left|\partial_{x}^{k}\left(\left(\bar{n}_{1}-n_{+}\right) \varphi_{1 x}\right)_{x}\right|+\left|\partial_{x}^{k}\left(\left(\bar{n}_{1}-n_{+}\right) \varphi_{2 x}\right)_{x}\right|\right. \\
& +\left|\partial_{x}^{k} \hat{n}_{1 x}\right|+\left|\partial_{x}^{k}\left(\varphi_{1 x}^{2}\right)_{x}\right|+\left|\partial_{x}^{k}\left(\varphi_{2 x}^{2}\right)_{x}\right|+\left|\partial_{x}^{k} \varphi_{1 x t} \varphi_{1 x}\right|+\left|\partial_{x}^{k} \varphi_{1 t}^{2} \bar{n}_{1 x}\right|+\left|\partial_{x}^{k} \varphi_{2 x t} \varphi_{2 x}\right| \\
& +\left|\partial_{x}^{k} \varphi_{2 t}^{2} \bar{n}_{1 x}\right|+\left|\partial_{x}^{k} \varphi_{1 t}^{2} \varphi_{1 x x}\right|+\left|\partial_{x}^{k} \varphi_{2 t}^{2} \varphi_{2 x x}\right|+\left|\partial_{x}^{k}\left(\left(\varphi_{1}+\varphi_{2}\right)_{x} E\right)\right| .
\end{aligned}
$$

From (2.9), (2.10), and (3.12)-(3.15), and by Holder's inequality, then the $L^{1}$-norm for $F$ can be estimated as follows

$$
\|F\|_{L^{1}\left(\mathbb{R}_{+}\right)} \leq C(1+t)^{-5 / 4}+C e^{-\alpha t} .
$$

Similarly, we can also prove

$$
\|F\|_{k} \leq C(1+t)^{-3 / 2}+C e^{-\alpha t}
$$

By noting (4.8), (4.9) and $3 / 2>5 / 4 \geq(2 k+1) / 4$ for $k=0,1,2$, and applying Lemmas 2.2 and 2.3 , we obtain optimal rates for the last term of (4.5) as follows

$$
\begin{aligned}
& \int_{0}^{t}\left\|\partial_{x}^{k} \int_{0}^{\infty}\left(K_{1}(x-y, t-\tau)-K_{1}(x+y, t-\tau)\right) F(y, \tau) d y\right\|_{L^{2}\left(\mathbb{R}_{+}\right)} d \tau \\
\leq & C \int_{0}^{t}(1+t-\tau)^{-(2 k+1) / 4}\left(\|F\|_{L^{1}\left(\mathbb{R}_{+}\right)}+\|F\|_{k}\right) d \tau \\
\leq & C \int_{0}^{t}(1+t-\tau)^{-(2 k+1) / 4}\left((1+t)^{-5 / 4}+(1+t)^{-3 / 2}+e^{-\alpha t}\right) d \tau \\
\leq & C(1+t)^{-(2 k+1) / 4} .
\end{aligned}
$$

Applying (4.6), (4.7) and (4.10) to (4.5), we have

$$
\left\|\partial_{x}^{k}\left(\varphi_{1}+\varphi_{2}\right)\right\|_{L^{2}\left(\mathbb{R}_{+}\right)} \leq C(1+t)^{-\frac{2 k+1}{4}}, \quad k=0,1,2 .
$$

Moreover, recall that

$$
\left\|\partial_{x}^{k}\left(\varphi_{1}-\varphi_{2}\right)\right\|_{L^{2}\left(\mathbb{R}_{+}\right)} \leq C e^{-\alpha t}, \quad k=0,1,2 .
$$

Therefore, (4.11), (4.12) and the triangle inequality lead to (4.1).

Now, we are going to prove (4.2). It is well known that

$$
\begin{aligned}
& \left(z_{1}+z_{2}\right)(x, t)=\left(\varphi_{1}+\varphi_{2}\right)_{t}(x, t) \\
= & \partial t \int_{0}^{\infty}\left(K_{0}(x-y, t)-K_{0}(x+y, t)\right)\left(\varphi_{10}+\varphi_{20}\right)(y) d y \\
& +\partial t \int_{0}^{\infty}\left(K_{1}(x-y, t)-K_{1}(x+y, t)\right)\left(z_{10}+z_{20}\right)(y) d y \\
& +\int_{0}^{t} \partial t \int_{0}^{\infty}\left(K_{1}(x-y, t-\tau)-K_{1}(x+y, t-\tau)\right) F(y, \tau) d y d \tau \\
& +\int_{0}^{\infty}\left(K_{1}(x-y, 0)-K_{1}(x+y, 0)\right) F(y, t) d y .
\end{aligned}
$$


By making use of the fashion as before, then Lemmas 2.2 and 2.3 help us to reach the goal

$$
\begin{aligned}
& \left\|z_{1}+z_{2}\right\|_{L^{2}\left(\mathbb{R}_{+}\right)}=\left\|\left(\varphi_{1}+\varphi_{2}\right)_{t}\right\|_{L^{2}\left(\mathbb{R}_{+}\right)} \\
& =\partial t \int_{0}^{\infty}\left(K_{0}(x-y, t)-K_{0}(x+y, t)\right)\left(\varphi_{10}+\varphi_{20}\right)(y) d y \|_{L^{2}\left(\mathbb{R}_{+}\right)} \\
& +\left\|\partial t \int_{0}^{\infty}\left(K_{1}(x-y, t)-K_{1}(x+y, t)\right)\left(z_{10}+z_{20}\right)(y) d y\right\|_{L^{2}\left(\mathbb{R}_{+}\right)} \\
& +\int_{0}^{t}\left\|\partial t \int_{0}^{\infty}\left(K_{1}(x-y, t-\tau)-K_{1}(x+y, t-\tau)\right) F(y, \tau) d y\right\|_{L^{2}\left(\mathbb{R}_{+}\right)} d \tau \\
& +\left\|\int_{0}^{\infty}\left(K_{1}(x-y, 0)-K_{1}(x+y, 0)\right) F(y, t) d y\right\|_{L^{2}\left(\mathbb{R}_{+}\right)} \\
& \leq C\left(\left\|\varphi_{10}+\varphi_{20}\right\|_{L^{1}\left(\mathbb{R}_{+}\right)}+\left\|\varphi_{10}+\varphi_{20}\right\|_{3}\right)(1+t)^{-5 / 4} \\
& +C\left(\left\|z_{10}+z_{20}\right\|_{L^{1}\left(\mathbb{R}_{+}\right)}+\left\|z_{10}+z_{20}\right\|_{2}\right)(1+t)^{-5 / 4} \\
& +C \int_{0}^{t}(1+t-\tau)^{-5 / 4}\left(\|F\|_{L^{1}\left(\mathbb{R}_{+}\right)}+\|F\|_{2}\right) d \tau \\
& +C\left(\|F\|_{L^{1}\left(\mathbb{R}_{+}\right)}+\|F\|_{2}\right) \\
& \leq C\left(\left\|\varphi_{10}+\varphi_{20}\right\|_{L^{1}\left(\mathbb{R}_{+}\right)}+\left\|\varphi_{10}+\varphi_{20}\right\|_{3}\right)(1+t)^{-5 / 4} \\
& +C\left(\left\|z_{10}+z_{20}\right\|_{L^{1}\left(\mathbb{R}_{+}\right)}+\left\|\bar{z}_{10}+z_{20}\right\|_{2}\right)(1+t)^{-5 / 4} \\
& +C \int_{0}^{t}(1+t-\tau)^{-5 / 4}\left((1+t)^{-5 / 4}+(1+t)^{-3 / 2}+e^{-\alpha t}\right) d \tau \\
& +C\left((1+t)^{-5 / 4}+(1+t)^{-3 / 2}+e^{-\alpha t}\right) \\
& \leq C(1+t)^{-5 / 4} \text {. }
\end{aligned}
$$

On the other hand, (3.14) gives

$$
\left\|z_{1}-z_{2}\right\|_{L^{2}\left(\mathbb{R}_{+}\right)}=\left\|\varphi_{1}-\varphi_{2}\right\|_{L^{2}\left(\mathbb{R}_{+}\right)}=\left\|E_{t}\right\|_{L^{2}\left(\mathbb{R}_{+}\right)} \leq C E^{-\alpha t} .
$$

Combining (4.14) and (4.15), and using the triangle inequality, we can obtain (4.2).

\section{Acknowledgements}

The author is grateful to the anonymous referees for careful reading and valuable comments which led to an important improvement of my original manuscript. The research is partially supported by the National Science Foundation of China (Grant No. 11171223).

\section{Competing interests}

The authors declare that they have no competing interests.

Received: 26 May 2011 Accepted: 16 February 2012 Published: 16 February 2012

\section{References}

1. Jüngel, A: Quasi-hydrodynamic semiconductor equations. Progress Nonlinear Diff Equ. 41, 191-196 (2001)

Markowich, PA, Ringhofev, CA, Schmeiser, C: Semiconductor Equations. Springer-Verlag, New York (1990)

Sitnko, A, Malnev, V: Plasma Physics Theory. Chapman \& Hall, London (1995) 
4. Zhou, F, Li, YP: Existence and some limits of stationary solutions to a one-dimensional bipolar Euler-Poisson system. J Math Anal Appl. 351, 480-490 (2009). doi:10.1016/j.jmaa.2008.10.032

5. Tsuge, $\mathrm{N}$ : Existence and uniqueness of stationary solutions to a one-dimensional bipolar hydrodynamic models of semiconductors. Nonlinear Anal TMA. 73, 779-787 (2010). doi:10.1016/j.na.2010.04.015

6. Natalini, R: The bipolar hydrodynamic model for semiconductors and the drift-diffusion equation. J Math Anal Appl. 198, 262-281 (1996). doi:10.1006/jmaa.1996.0081

7. Hsiao, L, Zhang, KJ: The relaxation of the hydrodynamic model for semiconductors to the drift-diffusion equations. J Diff Equ.165, 315-354

8. Hsiao, L, Zhang, K: The global weak solution and relaxation limits of the initial boundary value problem to the bipolar hydrodynamic model for semiconductors. Math. Models Methods Appl Sci. 10, 1333-1361 (2000). doi:10.1142/ S0218202500000653

9. Zhu, C, Hattori, H: Stability of steady state solutions for an isentropic hydrodynamic model of semiconductors of two species. J Diff Equ. 166, 1-32 (2000). doi:10.1006/jdeq.2000.3799

10. Ali, $G$, Jüngel, A: Global smooth solutions to the multi-dimensional hydrodynamic model for two-carrier plasma. J Diff Equ. 190, 663-685 (2003). doi:10.1016/50022-0396(02)00157-2

11. Lattanzio, C: On the 3-D bipolar isentropic Euler-Poisson model for semiconductors and the drift-diffusion limit. Math Models Methods Appl Sci. 10, 351-360 (2000)

12. Li, YP: Diffusion relaxation limit of a bipolar isentropic hydrodynamic model for semiconductors. J Math Anal Appl. 336, 1341-1356 (2007). doi:10.1016/j.jmaa.2007.03.068

13. Gasser, I, Marcati, P: The combined relaxation and vanishing Debye length limit in the hydrodynamic model for semiconductors. Math Methods Appl Sci. 24, 81-92 (2001). doi:10.1002/1099-1476(20010125)24:23.0.CO;2-X

14. Gasser, I, Hsiao, L, Li, HL: Large time behavior of solutions of the bipolar hydrodynamical model for semiconductors. J Diff Equ. 192, 326-359 (2003). doi:10.1016/S0022-0396(03)00122-0

15. Huang, FM, Li, YP: Large time behavior and quasineutral limit of solutions to a bipolar hydrodynamic model with large data and vacuum. Discr Contin Dyn Syst. A24, 455-470 (2009)

16. Marcati, P, Mei, M, Rubino, B: Optimal convergence rates to diffusion waves for solutions of the hyperbolic conservation laws with damping. J Math Fluid Mech. 7, 224-240 (2005). doi:10.1007/s00021-005-0155-9

17. Marcati, P, Mei, M: Convergence to nonlinear diffusion waves for solutions of the initial boundary problem to the hyperbolic conservation laws with damping. Quart Appl Math. 56, 763-784 (2000)

18. Nishihara, $K$, Wang, WK, Yang, $T: L^{p}$-convergence rate to nonlinear diffusion waves for $p$-system with damping. J Diff Equ. 161, 191-218 (2000). doi:10.1006/jdeq.1999.3703

19. Wang, WK, Yang, T: Pointwise estimates and $L^{p}$ convergence rates to diffusion waves for $p$-system with damping. J Diff Equ. 187, 310-336 (2003). doi:10.1016/50022-0396(02)00056-6

20. Matsumura, A: On the asymptotic behavior of solutions of semilinear wave equation. Publ RIMS Kyoto Univ. 12, 169-189 (1976). doi:10.2977/prims/1195190962

21. Zhao, HJ: Convergence to strong nonlinear diffusion waves for solutions of $p$-system with damping. J Diff Equ. 174, 200-236 (2001). doi:10.1006/jdeq.2000.3936

22. Zhu, CJ: Asymptotic behavio of solutions of p-system with relaxation. J Diff Equ. 180, 273-306 (2002). doi:10.1006/ jdeq.2001.4063

doi:10.1186/1687-2770-2012-21

Cite this article as: Li: Global existence and asymptotic behavior of smooth solutions for a bipolar Euler-Poisson system in the quarter plane. Boundary Value Problems 2012 2012:21.

\section{Submit your manuscript to a SpringerOpen ${ }^{\circ}$ journal and benefit from:}

- Convenient online submission

- Rigorous peer review

- Immediate publication on acceptance

- Open access: articles freely available online

- High visibility within the field

- Retaining the copyright to your article

Submit your next manuscript at $\$$ springeropen.com 